\title{
Relationship between Behavioral Activation and Inhibition Systems with Experiential Avoidance and Impulsivity of Veterans with Post-traumatic Stress Disorder
}

\section{ART ICLE INF O}

\section{Article Type}

Descriptive Study

\section{Authors}

Atadokht A. ${ }^{1} \mathrm{PhD}$,

Einy S.* $M S c$,

Tagavy R. ${ }^{2} M S C$

How to cite this article
Atadokht A, Einy S, Tagavy R. Re-
lationship between Behavioral
Activation and Inhibition Systems
with Experiential Avoidance and
Impulsivity of Veterans with Po-
st-traumatic Stress Disorder. Ira-
nian Journal of War \& Public He-
alth. 2018;10(2):69-74.

*Psychology Department, Educational Sciences \& Psychology Faculty, Mohaghegh Ardabili University, Ardabil, Iran

${ }^{1}$ Psychology Department, Educational Sciences \& Psychology Faculty, Mohaghegh Ardabili University, Ardabil, Iran

${ }^{2}$ Psychology Department, Psychology Faculty, Ardabil Branch, Islamic Azad University, Ardabil, Iran

\section{Correspondence}

Address: Psychology \& Educational Sciences Faculty, University of Mohaghegh Ardabili, Daneshgah Street, Ardabil, Iran

Phone: +98 (45) 33262678

Fax: +98 (45) 33511508

sanaz.einy@yahoo.com

\section{Article History}

Received: September 27, 2017

Accepted: January 11, 2017

ePublished: April 10, 2018

\section{A B S T R A C T}

Aims The sensitivity of behavioral activation and inhibition systems can play a role in the continuation of post-traumatic stress disorder (PTSD) in veterans. Regarding the importance of this issue, the present study was conducted with the aim of predicting the impulsivity and experiential avoidance of veterans with post-traumatic stress disorder based on the activation and behavioral inhibition systems.

Instruments \& Methods In the present descriptive correlational study, 120 veterans with post-traumatic stress disorders, hospitalized in Isar Psychiatric Hospital in Ardabil were selected, using available sampling method in 2017. The data were collected, using Behavioral Activation and Inhibition Systems Scale, Impulsivity questionnaire, and Acceptance and Action questionnaire. The data were analyzed by SPSS 21 software, using Pearson correlation coefficient and multiple regression analysis

Findings A total of $65.4 \%$ of the experiential avoidance variance and $79.1 \%$ of the impulsivity variance of veterans with PTSD were determined by behavioral activation and inhibition systems $(p=0.001)$. Behavioral inhibition system $(\beta=0.167)$ and sensitivity to reward $(\beta=0.66)$ predicted experiential and avoidance significantly $(\mathrm{p}<0.001)$. Also, sensitivity to reward $(\beta=0.366)$, directly and significantly, and behavioral inhibition $(\beta=-0.132)$, inversely and significantly, predicted the impulsivity of veterans with PTSD $(\mathrm{p}<0.001)$.

Conclusion The sensitivity of the behavioral activation and inhibition systems increases the experiential avoidance and impulsivity in the veterans with PTSD. Thus, by reducing the activity of brain/behavioral systems, it is possible to modulate experiential avoidance, impulsivity, and PTSD symptoms in veterans.

Keywords Behavioral Activation and Inhibition Systems; Experiential Avoidance; Impulsivity; Post-traumatic Stress Disorder ; Veteran

\section{I T A T I O N L I N K S}

[1] The effect of cognitive-behavioral couple ... [2] Diagnostic and statistical manual of mental ... [3] Evaluation of post-traumatic stress disorder ... [4] The role of experiential avoidance in ... [5] Anhedonia in the daily lives of depressed ... [6] Acceptance and commitment therapy for ... [7] Coping and experiential avoidance ... [8] Emotion regulation in action: Emotional reactivity ... [9] Experiential avoigance and the relationship between ... [10] The role of experiential avoidance and mindfulness ... [11] Differential effects of anxiety sensitivity components in the ... [12] The interactive effect of cognitive fusion and ... [13] Pre-deployment trait anxiety, anxiety sensitivity ... [14] Risk-taking behaviors and impulsivity ... [15] Personality Trait ... [16] Impulsivity and adolescent substance ... [17] Cognitive and behavioral inhibition in ... [18] Impulsivity is relevant for trauma ... [19] A preliminary investigation of the ... [20] Emotion regulation difficulties in trauma ... [21] The effect of anger management skill training ... [22] Rapid-response impulsivity predicts ... [23] The neuropsychology of anxiety ... [24] Comparison study of behavioral ..

[25] Behavioral inhibi-tion and PTSD ... [26] Experiential a voidance as a moderatore

[27] PTSD's underlying symptom dimensions ... [28] Theoretical and practical research ...

[29] Behavioral inhibition, behavioral activation ... [30] Relationships between child and ...

[31] Validity and reliability of behavioral ... [32] The relationship between behavioral ... [33] Neuropsychological and cognitive psychophysiological substrates of ... [34] Factor structure of the Barratt impulsiveness ... [35] Psychometric properties of an Iranian version of the Barratt impulsiveness ... [36] Preliminary psychometric properties of the ... [37] Psychometric properties of Persian version ... [38] Experiential avoidance mediates the ... [39] Impulsive behaviors as an emotion regulation strategy ... 
است كه مىتواند يس از مواجهه با يك رويداد آسيبزا ايجاد شود

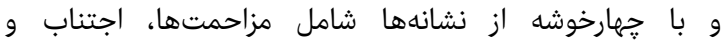

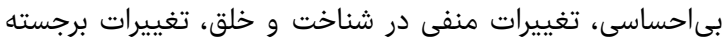

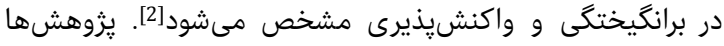

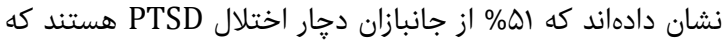

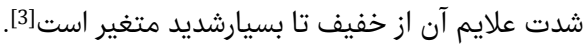

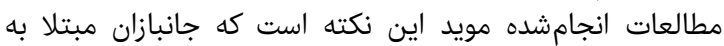

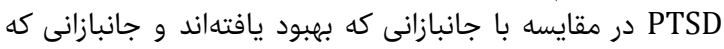

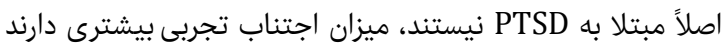

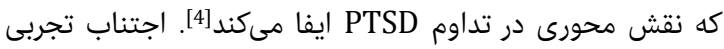

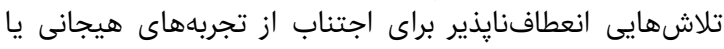

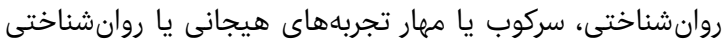

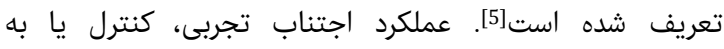

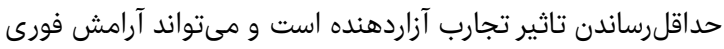

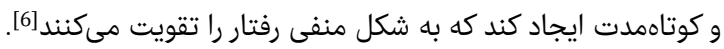

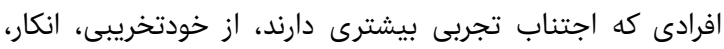

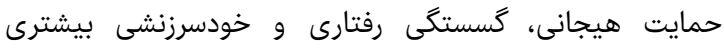

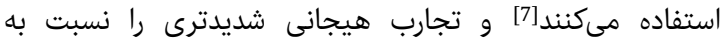

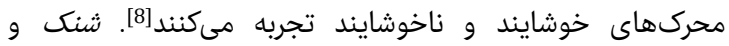

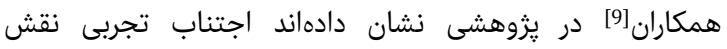

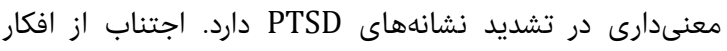

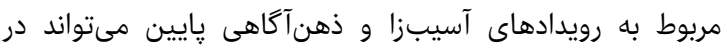

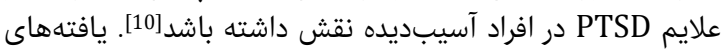

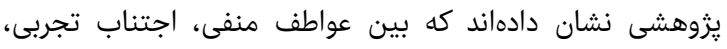

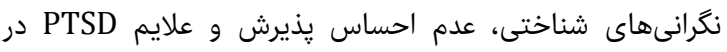

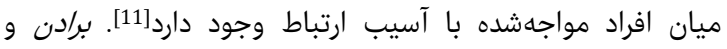

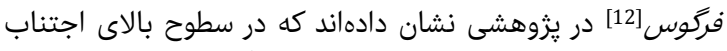

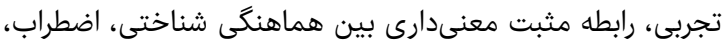

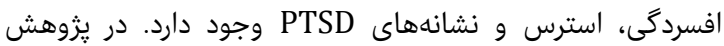

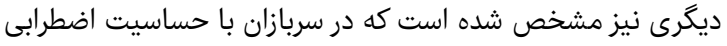

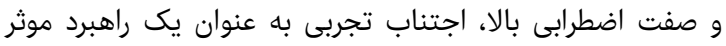

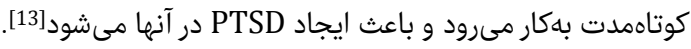

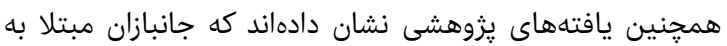

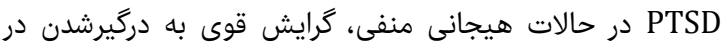

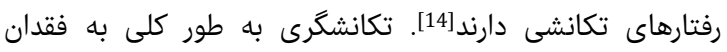

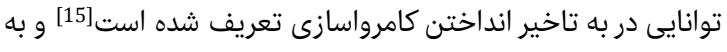

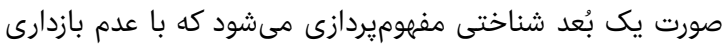

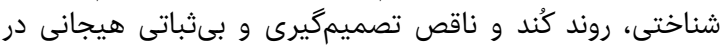

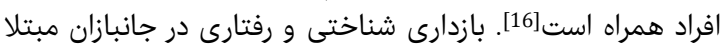

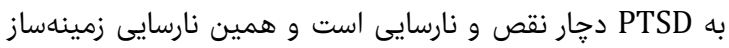

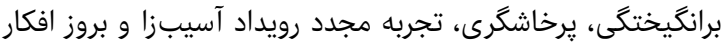

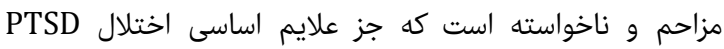

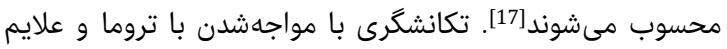

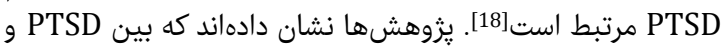

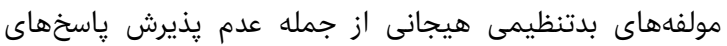

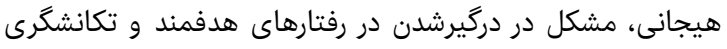

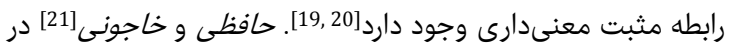

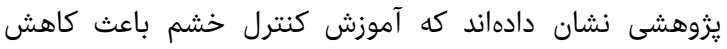

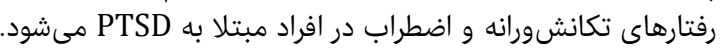

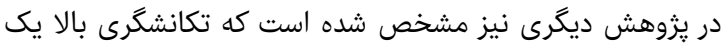

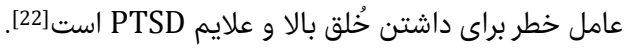

رابطه سيستمهاى فعالسازى و بازدارى رفتارى بال

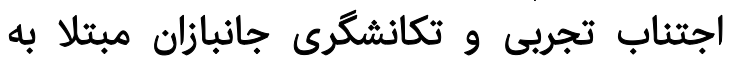
اختلال استرس يس از سانحه

اكبر عطادخت PhD گروه روانشناسى، دانشكده علومتربيتى و روانشناسى، دانشگاه محقق اردبيلى، اردبيل، ايران

MSc " ساناز عينى كروه روانشناسى، دانشكده علومتربيتى و روانشناسى، دانشگاه محقق اردبيلى، اردبيل، ايران

MSc رامين تقوى

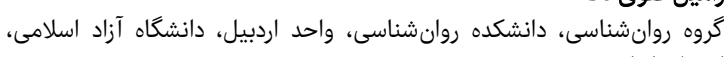
اردبيل، ايران

جكيده اهذاف: حساسيت سيستمهاى فعالسازى و بازدارى رفتارى مىتواند در تداوم

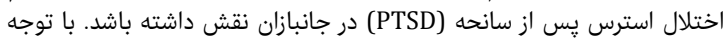

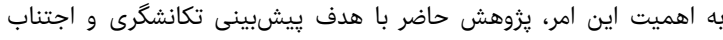

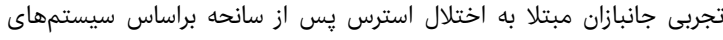

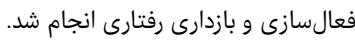

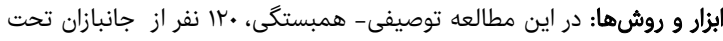

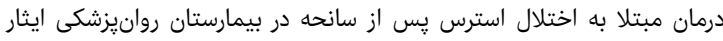

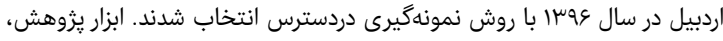

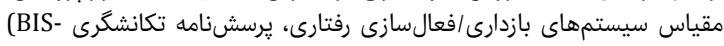

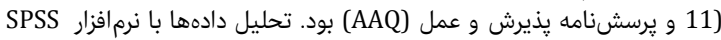

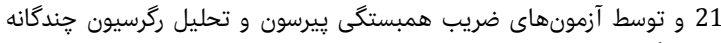

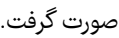

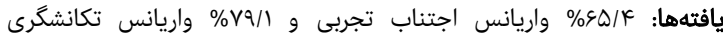

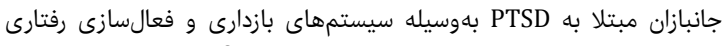

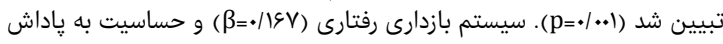

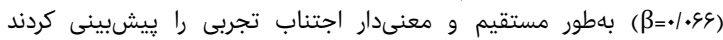

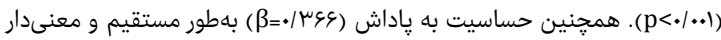

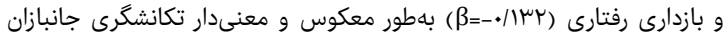

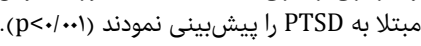

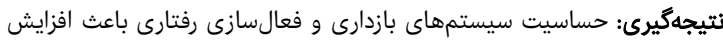

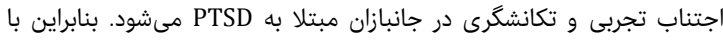

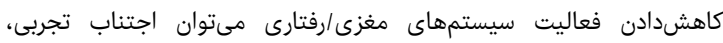

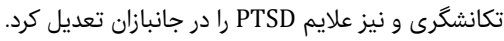

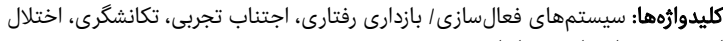
استرس ڤֶ از سانحه، جانباز

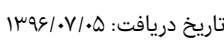

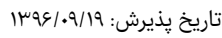

sanaz.einy@yahoo.com : نويسنده مسئول:

مقدمه

عوارض و ييامدهاى ناشى از جنگ به عند عنوان مهمترين مباحث

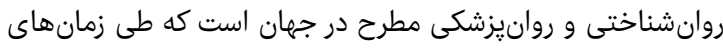

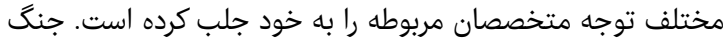

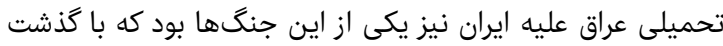

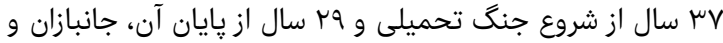

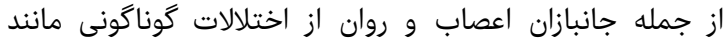

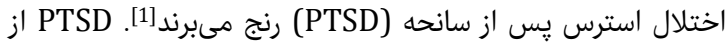

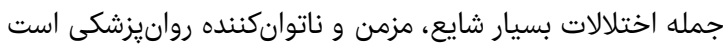

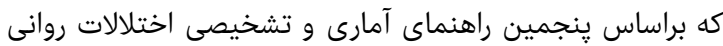

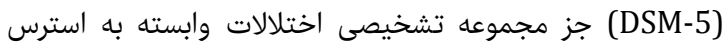




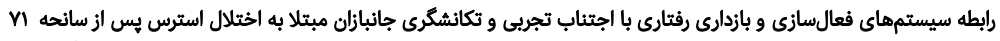

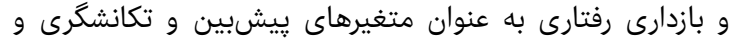

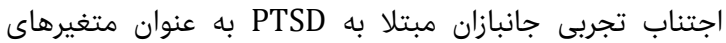

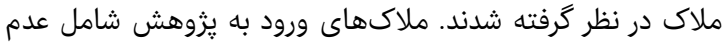

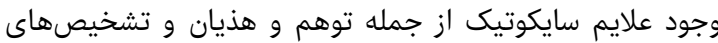

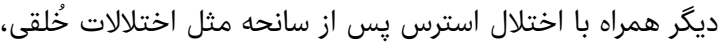

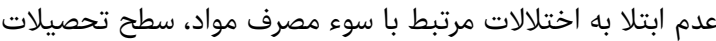

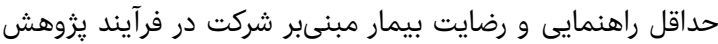

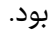

يرسشنامههاى ذيل، به عنوان ابزار يزوهش استفاده شده است.

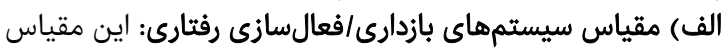

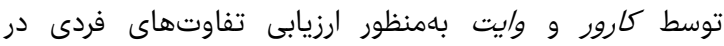

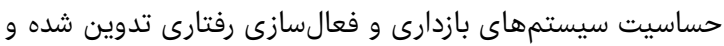

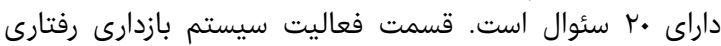

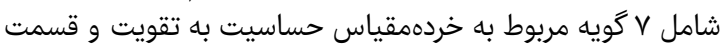

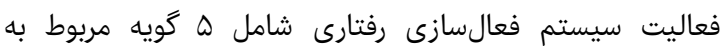

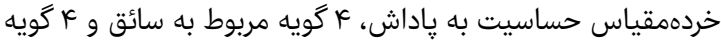

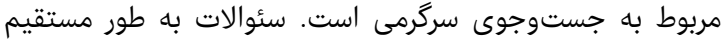

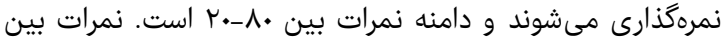

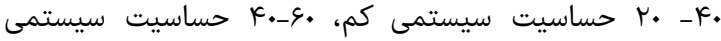

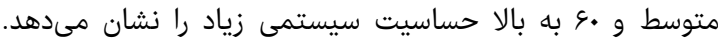

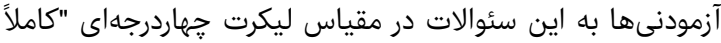

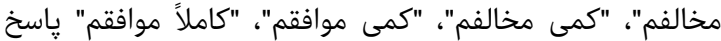

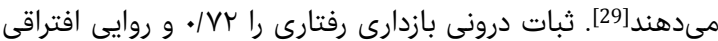

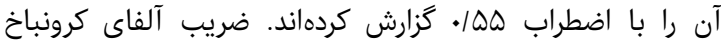

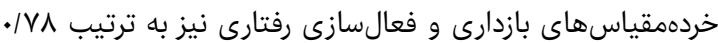

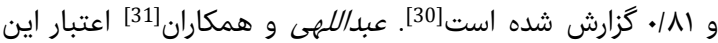

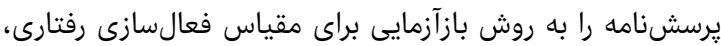

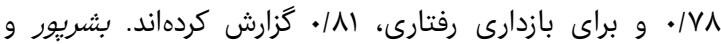

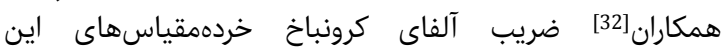

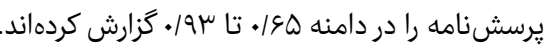
ب) برسشنامه تكانشگرى (BIS-11): نسخه يازدهم مقياس

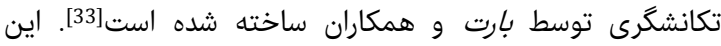

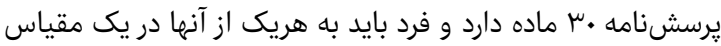

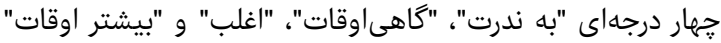

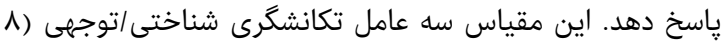

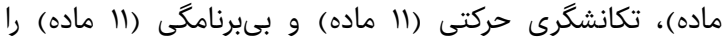

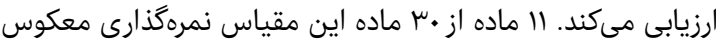

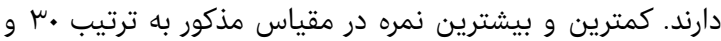

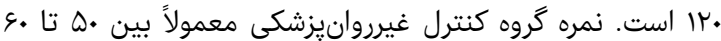

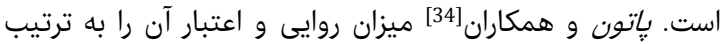
1AV محاسبه ضريب همبستگى زير مقياسهاء

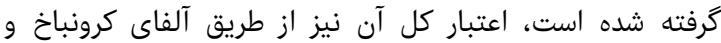

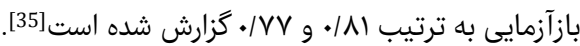

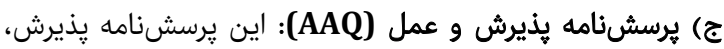

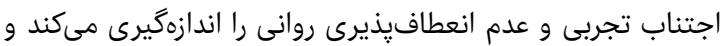

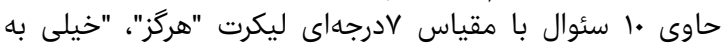

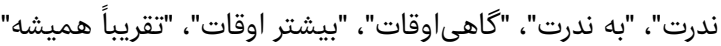

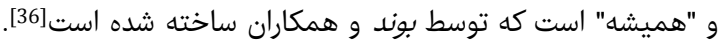

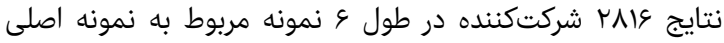

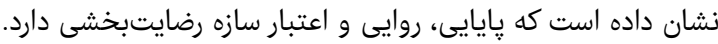

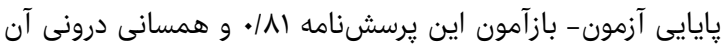

يكى از نظريههايى كه مىتوان براى توصيف و تبيين تفاوتهاى

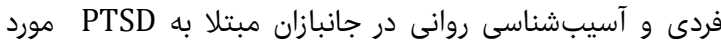
توجه قرار داد، نظريه حساسيت به تقويت (RST) كرىى]

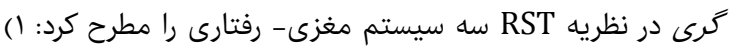

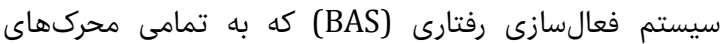

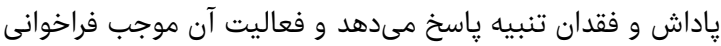

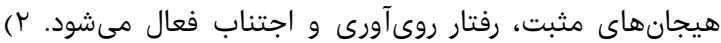

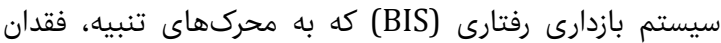

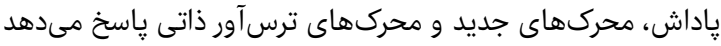

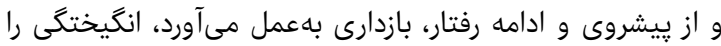

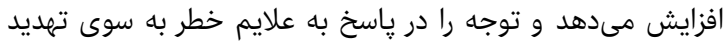

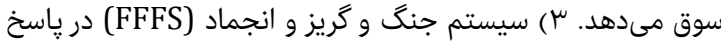

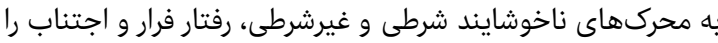

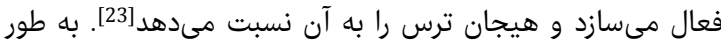

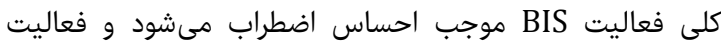

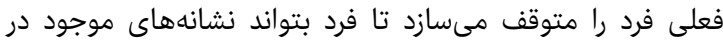

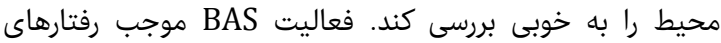

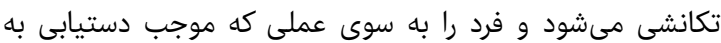

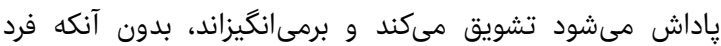

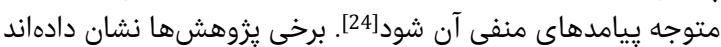

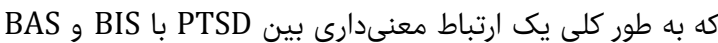

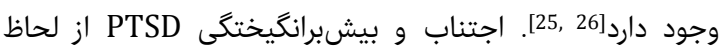

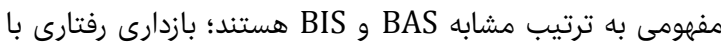

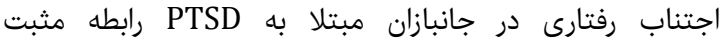

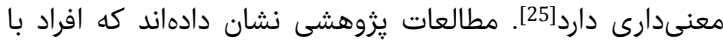
حساسيت بالا در BIS و اجتناب تجربى بالا، از افرادئ دادى كه

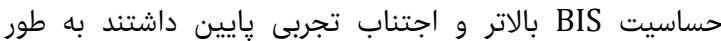
معنىدارى نشانههاى بيشترى از

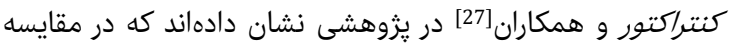

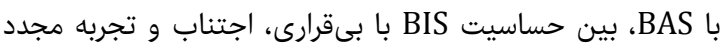

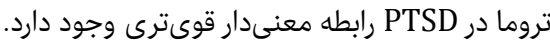

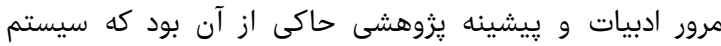

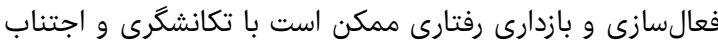

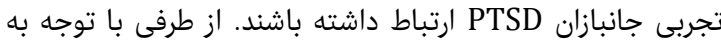

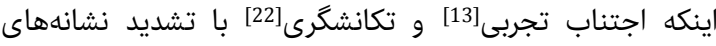

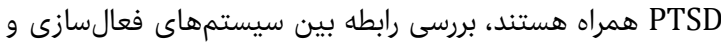

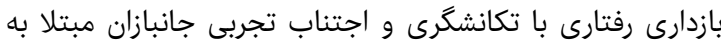
PTSD

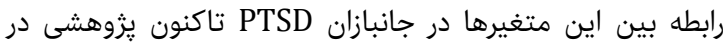

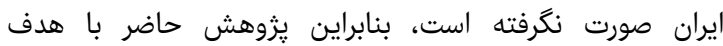

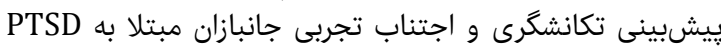

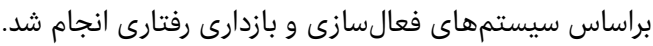

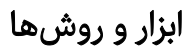

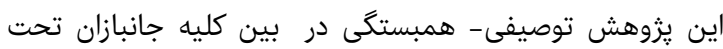

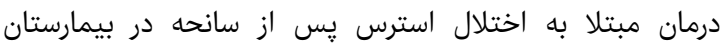

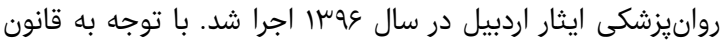

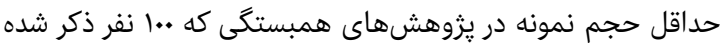

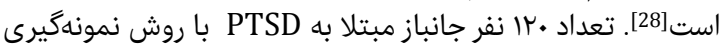

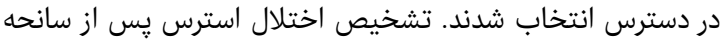

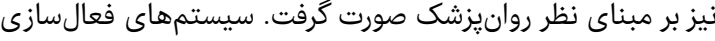


تحليل رگرسيون جندگانه براى تبيين اجتناب تجربى و تكانشكرى

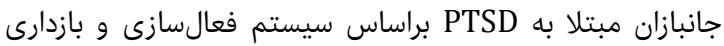

$$
\text { رفتارى صورت گرفت. }
$$

\section{يافتهها}

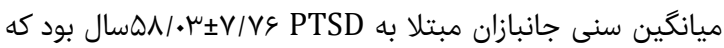

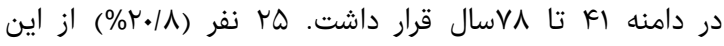

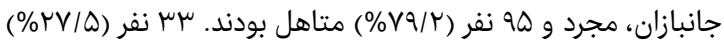

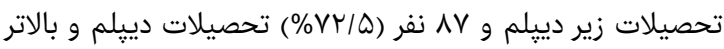

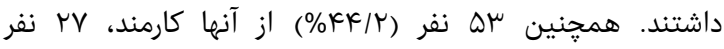

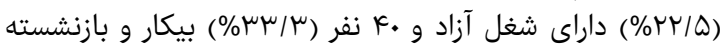

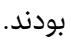

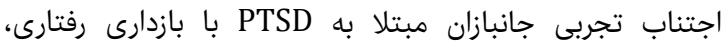

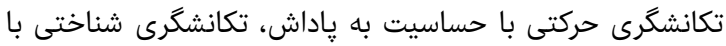

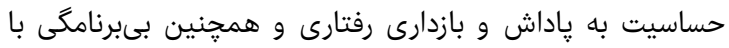

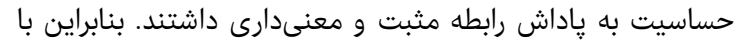

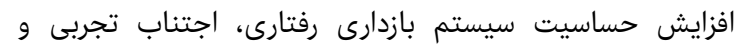

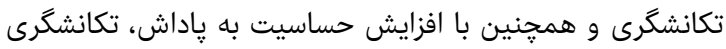

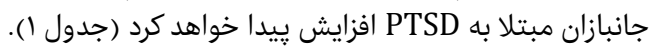

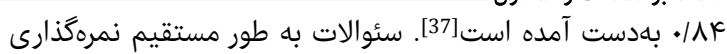

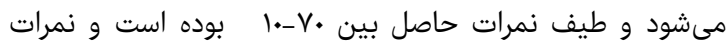

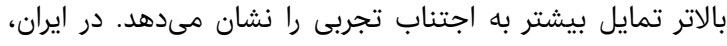

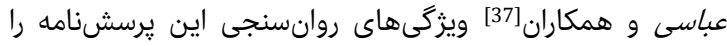

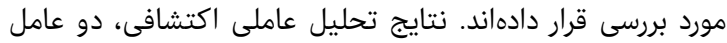

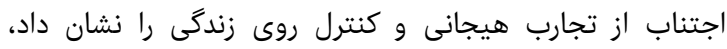

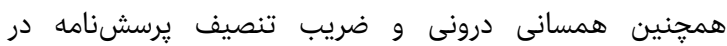

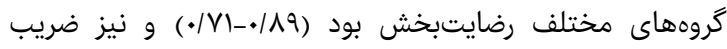

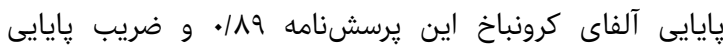

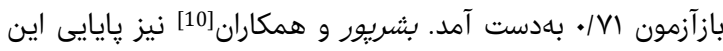

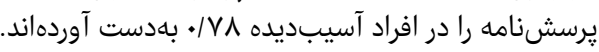

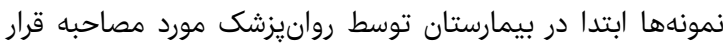

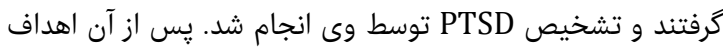

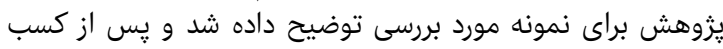

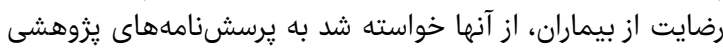
ياسخ دهند.

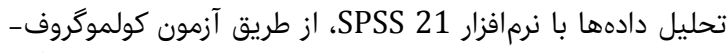

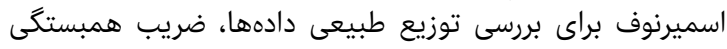

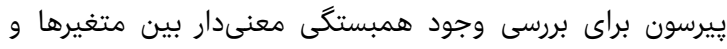

جدول () ميانكَين آمارى نمرات و ماتريس همبستخى اجتناب تجربى، مولفههاى تكانشكرى، سيستم بازدارى رفتارى و مولفههاى سيستم فعالسازى رفتارى در جانبازان مبتلا به نكانه

\begin{tabular}{|c|c|c|c|c|c|c|c|c|}
\hline$v$ & 8 & $\Delta$ & $k$ & $\mu$ & $r$ & 1 & نمرات & متغيرها \\
\hline & & & & & & 1 & $\mu V / V I \pm I \kappa / \kappa \mu$ & |-اجتناب تجربى \\
\hline & - & & & & 1 &.$/ V V$ & $r r / 1 \cdot \pm 9 / 1 K$ & r-تكانشكرى حركتى \\
\hline & & & & 1 & $\cdot / \wedge V^{* *}$ & $\cdot / V^{\mu}$ & $r / / \Delta q \pm \Lambda / Q \Lambda$ & س-تكانشكرى شناختى \\
\hline & & & 1 &.$/ 4 q^{*}$ &.$|\Delta|^{* *}$ & $\cdot / \Delta \Delta$ & $r K / K \Delta \pm V / I \varepsilon$ & 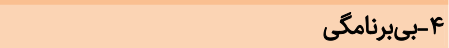 \\
\hline & & 1 &.$/ F \Delta^{* *}$ &.$/ \vee q^{* *}$ & $\cdot / \wedge \varsigma^{* *}$ &.$- / V T$ & $1.199 \pm \mu / \varepsilon F$ & ه-حساسيت به پاداش \\
\hline & 1 & $\cdot / 1 k$ & $\cdot 1 \cdot \Delta$ & $\cdot / \bullet Y$ & $\cdot / \cdot \mu$ & (. & $1 \cdot / \Lambda \Lambda \pm F / / \mu$ & 9-سائق 1 \\
\hline 1 &.$/ 4 \varphi^{*}$ & $\cdot / 1 \mu$ & $\cdot \mu^{\mu}$. & .1 .9 & $\cdot / 11$ & $\cdot / \cdot V \Delta$ & $q / \mu \mu \pm r / \wedge \Delta$ & V-جستوجوى سركرمى \\
\hline $.1 \cdot V$ &.$/ \cdot 1$ &.$/ A V^{*}$ & . $|8|$ & $\cdot \mid \Delta \mu^{*}$ &.$|k|$ & ./VQ** & $\mid Q / \Lambda \Lambda \pm \varepsilon / V \varepsilon$ & ^-بازدارى رفتارىى \\
\hline
\end{tabular}

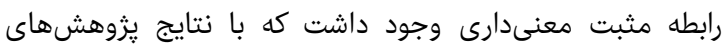

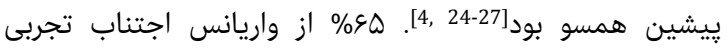

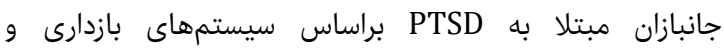

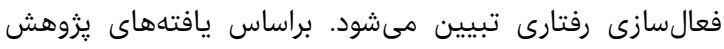

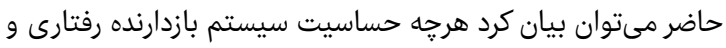

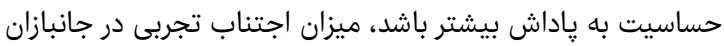

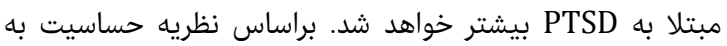

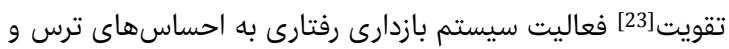

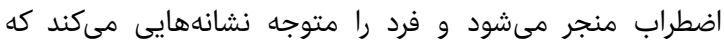

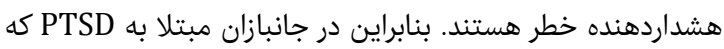

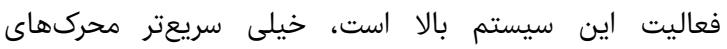

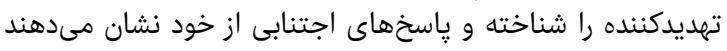

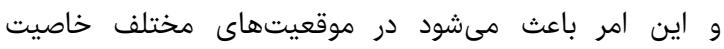

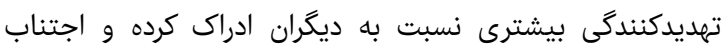

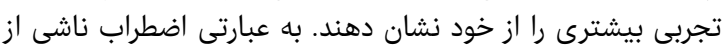

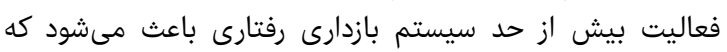

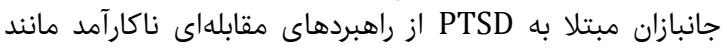

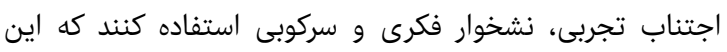

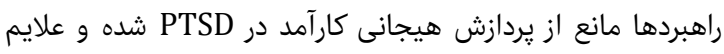

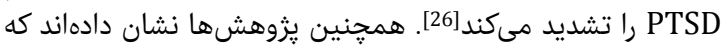

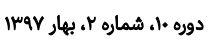

سيستم بازدارى و فعالسازى رفتارى، ييشبينى إكننده خوبى براى

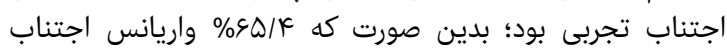

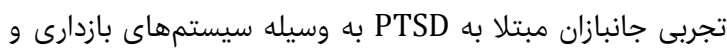

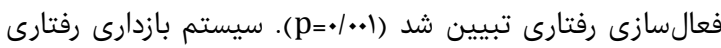

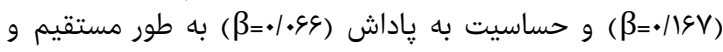
معنىدار اجتناب تجربى جانبازان مبتلا به

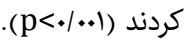

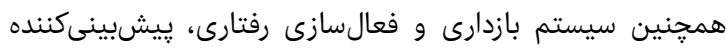

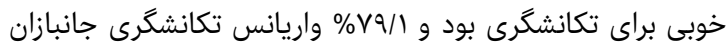

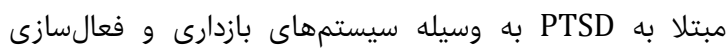

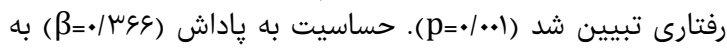

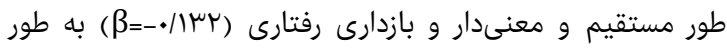

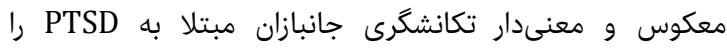

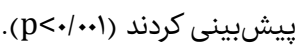

يزرهش حاضر با هدف ييشبينى تكانشگرى و اجتناب تجربى براب

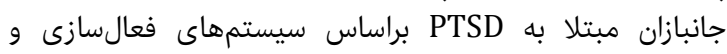

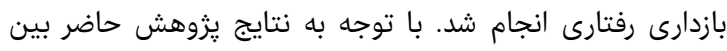

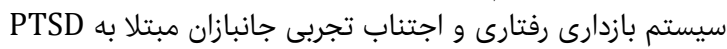




\section{نتيجهكيرى}

حساسيت سيستمهاى بازدارى و فعالسازى رفتارى باعث افزايش

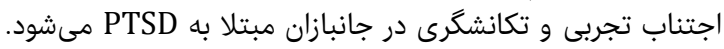

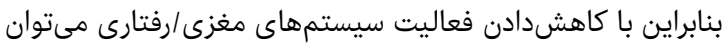

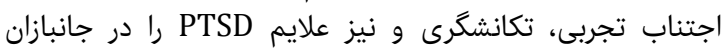

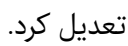

تشكر و قدردانى: نويسندكان اين يزوهش از همكارى جانبازان عزيز

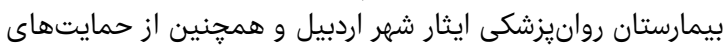

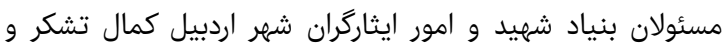

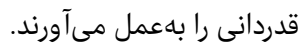

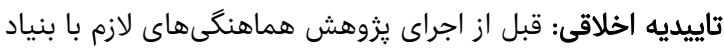

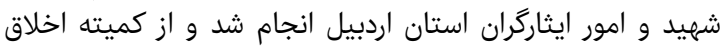

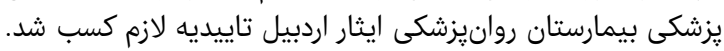

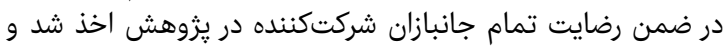

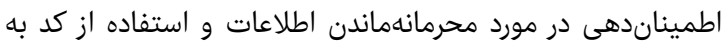

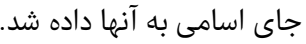
تعارض منافع: موردى از سوى نويسندكان بيان آنشان نشده است.

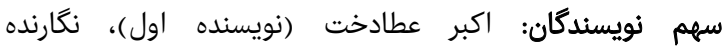

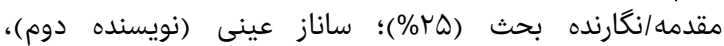

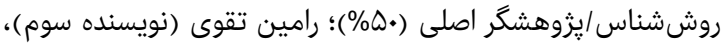

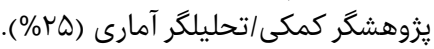

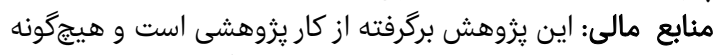
حمايت مالى از سوى نهاد يا سازمانى انجام نكرفته است.

منابع

1- Zooghi-Paydar MR. The effect of cognitive-behavioral couple therapy on post-traumatic stress disorder and depression in veterans. J Woman Fam Stud. 2013;1(1):99-118. [Persian]

2- American Psychiatric Association. Diagnostic and statistical manual of mental disorders DSM-5. $5^{\text {th }}$ Edition. Rezaei F, Fakhraee A, Farmand A, Niloofari A, Hashemi Azar J, Shamloo F, translators. Tehran: Arjmand; 2013. pp. 102-9. [Persian]

3- Kamalmanesh A, Maredpour A. Evaluation of posttraumatic stress disorder among war survivors. Health Res J. 2017;2(2):87-98. [Persian]

4- Morina N. The role of experiential avoidance in psychological functioning after war-related stress in Kosovar civilians. J Nerv Ment Dis. 2007;195(8):697-700. 5- Hershenberg R, Mavandadi S, Wright E, Thase ME. Anhedonia in the daily lives of depressed veterans: A pilot report on experiential avoidance as a moderator of emotional reactivity. J of Affect Disord. 2017;208:414-7.

6- Eifert GH, Forsyth JP, Arch J, Espejo E, Keller M, Langer D. Acceptance and commitment therapy for anxiety disorders: Three case studies exemplifying a unified treatment protocol. Cogn Behav Pract. 2009;16(4):36885.

7- Karekla M, Panayiotou G. Coping and experiential avoidance: Unique or overlapping constructs?. J Behav Ther Exp Psychiatry. 2011;42(2):163-70.

8- Sloan DM. Emotion regulation in action: Emotional reactivity in experiential avoidance. Behav Res Ther. 2004;42(11):1257-70.
قراركرفتن در معرض تروما و علايم PTSD با حساسيت BIS همراه

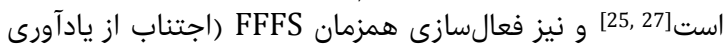

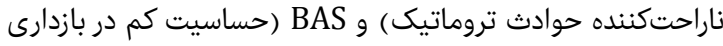

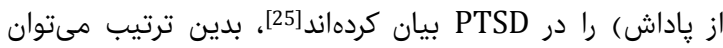

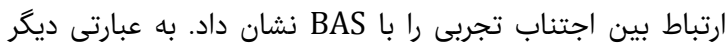

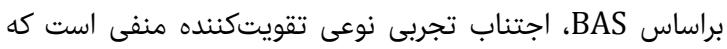

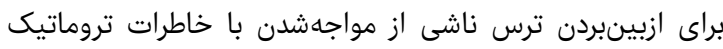

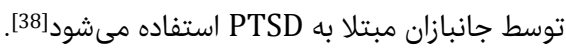

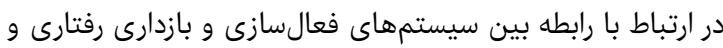

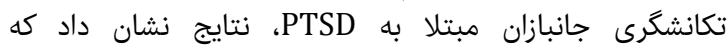

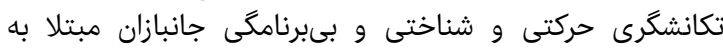

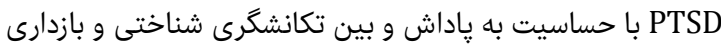

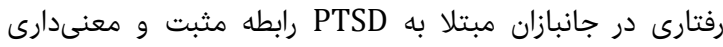

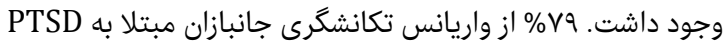

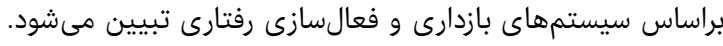

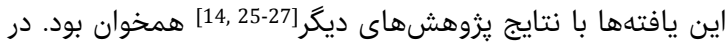

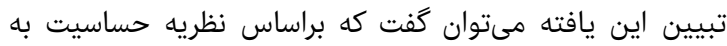
تقويت[23]، فعاليت بيش از حئ حد

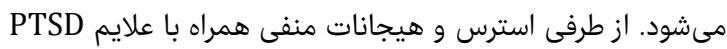

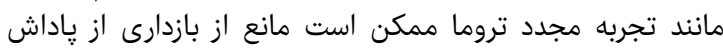

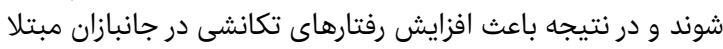

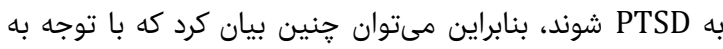

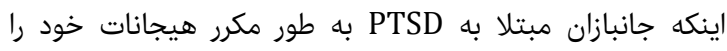

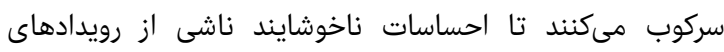

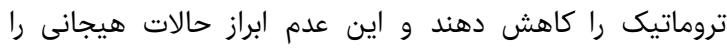

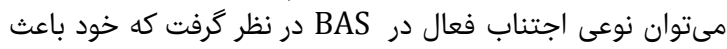

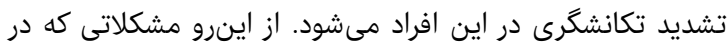

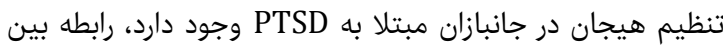

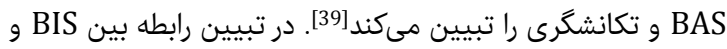

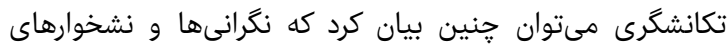

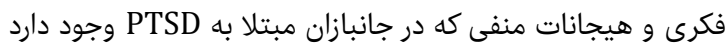

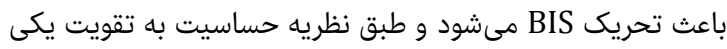

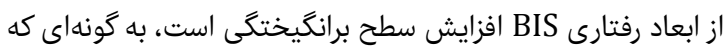

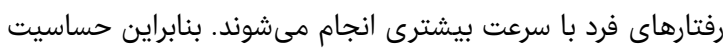

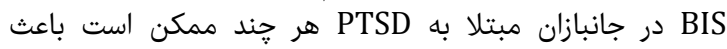

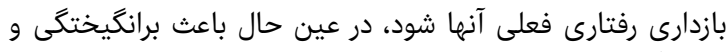

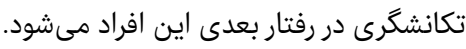

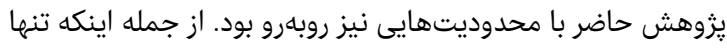

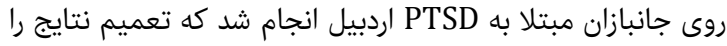

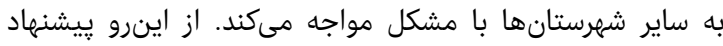

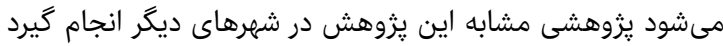

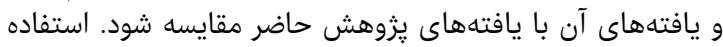

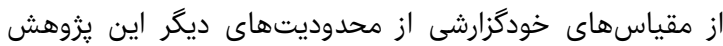

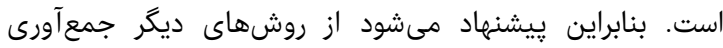

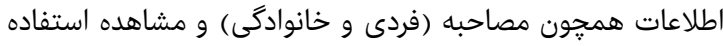

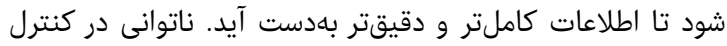

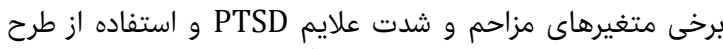

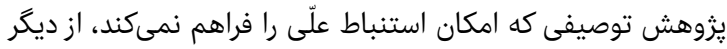

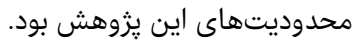


patients and normal individuals. J Babol Univ Med Sci. 2012;14(2):59-65. [Persian]

25- Myers CE, VanMeenen KM, Servatius, RJ. Behavioral inhibi-tion and PTSD symptoms in veterans. Psychiatry Res. 2012;196(2-3):271-6.

26- Pickett SM, Bardeen JR, Orcutt HK. Experiential a voidance as a moderatore of the relationship between behaviore inhibition system sensitivity and posttraumatic stress symptom. J Anxiety Disord. 2011;25(8):1038-46.

27- Contractor A, Elhai JD, Ractliffe KC, Forbes D. PTSD's underlying symptom dimensions and relations with behavioral inhibition and activation. J Anxiety Disord. 2013;27(7):645-51.

28- Delavar A. Theoretical and practical research in the humanities and social sciences. Teharn: Roshd; 2009. [Persian]

29- Carver CS, White TL. Behavioral inhibition, behavioral activation, and affective responses to impending reward and punishment: The BIS/BAS scales. J Personal Soc Psychol. 1994;67(2):319-33.

30- Muris P, Meesters C, Spinder M. Relationships between child-and parent-reported behavioural inhibition and symptoms of anxiety and depression in normal adolescents. Personal Individ Differ. 2005;34:759-71.

31- Abdollahi R, Bakhshipour R, Mahmoodaliloo M. Validity and reliability of behavioral inhibition and activation systems (BIS/BAS) scales among Tabriz university students. J Manag Syst. 2013;7(28):123-39. [Persian]

32- Basharpoor S, Barahmand U, Molavi P, Mousavi SA. The relationship between behavioral activation/inhibition systems (BAS/BIS) and bullying/victimization behaviors among male adolescents. Iran J Psychiatry. 2013;8(3):118-23.

33- Barratt ES, Stanford MS, Kent TA, Felthous A. Neuropsychological and cognitive psychophysiological substrates of impulsive aggression. Biol Psychiatry. 1997;41(10):1045-61.

34- Patton JH, Stanford MS, Barratt ES. Factor structure of the Barratt impulsiveness scale. J Clin Psychol. 1995;51(6):768-74.

35- Javid M, Mohammadi N, Rahim CH. Psychometric properties of an Iranian version of the Barratt impulsiveness scale-11 (BIS-11). J Manage Syst. 2012;(8):23-34. [Persian]

36- Bond FW, Hayes SC, Baer RA, Carpenter KM, Guenole $\mathrm{N}$, Orcutt HK et al. Preliminary psychometric properties of the Acceptance and Action Questionnaire-II: a revised measure of psychological inflexibility and experiential avoidance. Behav Ther. 2011;42(4):676-88.

37- Abbasi I, Fati L, Moludi R, Zarrabi H. Psychometric properties of Persian version of acceptance and action questionnaire-II. J Psychol Model Method. 2013;3(10):65-80. [Persian]

38- Maack DJ, Tull MT, Gratz KL. Experiential avoidance mediates the association between behavioral inhibition and post-traumatic stress disorder. Cogn Ther Res. 2011;36:407-16.

39- Weiss NH, Tull MT, Viana AG, Anestis MD, Gratz KL. Impulsive behaviors as an emotion regulation strategy: Examining associations between PTSD, emotion dysregulation, and impulsive behaviors among substance dependent inpatients. J Anxiety Disord. 2012;26(3):4538.
9- Shenk CE, Putnam FW, Noll JG. Experiential avoigance and the relationship between child maltreatment and PTSD symptom: Preliminary evidence. Child Abuse Negl. 2012;36(2):118-26.

10- Basharpoor S, Shafiei M, Atadokht A, Narimani M. The role of experiential avoidance and mindfulness in predicting the symptoms of stress disorder after exposure to trauma in traumatized people supported by Emdad Committee and Bonyade Shahid organization of Gilan Gharb in the First half of 2014. J Rafsanjan Univ Med Sci. 2015;14(5):405-16. [Persian]

11- Bakhshaie J, Zvolensky MJ, Allan N, Vujanovic AA, Schmidt NB. Differential effects of anxiety sensitivity components in the relation between emotional nonacceptance and post-traumatic stress symptoms among trauma-exposed treatment-seeking smokers. Cogn Behav Ther. 2015;44(3):175-89.

12- Bardeen JR, Fergus ThA. The interactive effect of cognitive fusion and experiential avoidance on anxiety, depression, stress and posttraumatic stress symptoms. J Context Behav Sci. 2016;5(1):1-6.

13- Cobb AR, Lancaster CL, Meyer EC, Lee HJ, Telch MJ. Pre-deployment trait anxiety, anxiety sensitivity and experiential avoidance predict war-zone stress-evoked psychopathology. J Context Behav Sci. 2017;6(3):276-87. 14- James LM, Strom TQ, Leskela J. Risk-taking behaviors and impulsivity among veterans with and without PTSD and mild TBI. Mil Med. 2014;179(4):357-63.

15- Basharpoor S. Personality Trait (theory and testing). $1^{\text {st }}$ Edition, Tehran: Savalan; 2015. [Persian]

16- Gullo MJ, Dawe S. Impulsivity and adolescent substance use: Rashly dismissed as "all-bad"?. Neurosci Biobehav Rev. 2008;32(8):1507-18.

17- Mirdoraghi F, Ghanbary Hashemabady B, Mashhadi A. Cognitive and behavioral inhibition in veterans withand without post-traumatic stress disorder. J Mil Med. 2012;14(1):41-7. [Persian]

18- Netto LR, Pereira JL, Nogueira JF, Cavalcanti-Ribeiro $\mathrm{P}$, Santana RC, Teles CA, et al. Impulsivity is relevant for trauma exposure and PTSD symptoms in a non-clinical population. Psychiatry Res. 2016;239:204-11.

19- Tull MT, Barrett HM, McMillan ES, Roemer L. A preliminary investigation of the relationship between emotion regulation difficulties and posttraumatic stress symptoms. Behav Ther. 2007;38(3):303-13.

20- Ehring T, Quack D. Emotion regulation difficulties in trauma survivors: The role of trauma type and PTSD symptom severity. Behav Ther. 2010;41(4):587-98.

21- Hafezi F, Khajuni A. The effect of anger management skill training on reduction of impulsive behaviors and anxiety in patients with PTSD. J Soc Psychol. 2012;5(20):89-107. [Persian]

22- Bjork JM, Burroughs TK, Franke LM, Pickett TC, Johns SE, Moeller FG, et al. Rapid-response impulsivity predicts depression and posttraumatic stress disorder symptomatology at 1-year follow-up in blast-exposed service members. Arch Phys Med Rehabil. 2017;98(8):1646-51.

23- Gray JA, McNaughton N. The neuropsychology of anxiety: An enquiry into the functions of the septohippocampus system. 2nd Edition. New York: Oxford University Press; 2000.

24- Kalantari H, Bagherian-Sararoudi R, Babaeipour E. Comparison study of behavioral activation system and behavioral inhibition system in irritable bowel syndrome 